\title{
Activation of the cAMP Pathway in Ustilago maydis Reduces Fungal Proliferation and Teliospore Formation in Plant Tumors
}

\author{
Julia Krüger, ${ }^{1}$ Gabriel Loubradou, ${ }^{1}$ Gerhard Wanner, ${ }^{2}$ Erika Regenfelder, ${ }^{1}$ Michael Feldbrügge, ${ }^{1}$ \\ and Regine Kahmann' \\ ${ }^{1}$ Institut für Genetik und Mikrobiologie der Universität München, Maria-Ward-Str.1a, D-80638 Munich, \\ Germany; 'Institut für Botanik der Universität München, Menzinger Str. 61-65, D-80638 Munich, Germany \\ Accepted 1 July 2000.
}

\begin{abstract}
In the corn smut fungus Ustilago maydis, mating of two haploid sporidia is a prerequisite for subsequent colonization of the host. Cyclic AMP (cAMP) and pheromone signals have been implicated in this developmental program. The cAMP pathway is also needed for subsequent fungal development in planta, as null mutants in any component of the pathway fail to form tumors. Here we show that moderate activation of the pathway conferred either by mutation in the $G \alpha$ subunit or by mutation in the regulatory subunit of the protein kinase A influences tumor morphology. In the resulting tumors, the amount of fungal material is drastically reduced and fungal development is arrested at the stage of sporogenic hyphae. We conclude that tight regulation of the CAMP pathway is crucial for fungal development within the plant but does not interfere with the tumor induction process.
\end{abstract}

Pathogenic microorganisms depend on the perception of environmental signals to induce developmental programs that are needed for colonization of the host. In the corn pathogen Ustilago maydis, mating of two haploid sporidia is a prerequisite for the formation of infection structures. Mating and subsequent pathogenic development are controlled by the biallelic $a$ mating type locus as well as the multiallelic $b$ locus (Banuett 1995). The $a$ locus codes for a lipopeptide pheromone precursor ( $m f a l / 2)$ and a receptor (pral/2) (Bölker et al. 1992). Binding of the pheromone to the receptor of the opposite $a$ mating type induces formation of mating hyphae and triggers cell fusion (Spellig et al. 1994). After cell fusion, the resulting dikaryon assumes hyphal growth. The $b$ gene products regulate pathogenic development through combinatorial interactions of a pair of homeodomain proteins, termed bW

Corresponding author: Regine Kahmann, Institut für Genetik und Mikrobiologie, Maria-Ward-Str.1a, D-80638 München, Germany; Telephone: +49-89-21806150; Fax: +49-89-1785633; E-mail: R.Kahmann@ lrz.uni-muenchen.de

Current address of Julia Krüger: Sainsbury Laboratory, Norwich Research Park, Colney Lane, Norwich NR4 7UH, U.K.

Both Julia Krüger and Gabriel Loubradou contributed equally to this work. and bE (Gillissen et al. 1992). bW and bE have been shown to form an active heterodimeric complex only if they are derived from different alleles (Kämper et al. 1995). Generation of such a heterodimeric complex is sufficient to trigger pathogenic development (Kämper et al. 1995). Diploid strains that are heterozygous at the $b$ locus or haploid strains expressing a pair of $b$ genes whose products can dimerize are termed solopathogenic, because they can infect the host without a prior mating event. The central regulator of the genes in the $a$ and $b$ locus is the pheromone response factor Prf1 (Hartmann et al. 1996). We have shown recently that its activity is influenced by the pheromone as well as the cyclic AMP (cAMP) signaling cascade (Müller et al. 1999; Hartmann et al. 1999; Krüger et al. 1998). The components of the cAMP pathway are the activating $\mathrm{G} \alpha$ subunit, Gpa3 (Regenfelder et al. 1997; Krüger et al. 1998), the adenylate cyclase, Uac1, and the regulatory and catalytic subunit of the protein kinase A (PKA), Ubc1 and Adr1, respectively (Gold et al. 1994, 1997). It has been shown that solopathogenic strains lacking any of these genes are unable to form tumors. While mutants in uacl, gpa3, and adrl fail to induce any symptoms, null mutants in $u b c l$, in which Adr1 is supposed to be fully active, are able to undergo the early steps of infection (Gold et al. 1997). In addition, a gpa3 allele, $g p a 3_{Q 206 L}$, has been described whose product should be compromised in its GTPase activity and represent a constitutively active form. In $g p a 3_{Q 206 L}$ strains, high amounts of pheromone gene expression are observed and in this respect such strains behave as strains in which $u b c l$ is deleted (Regenfelder et al. 1997; Krüger et al. 1998). This was taken as evidence that, in $\operatorname{gpa}_{Q 206 L}$ strains, the cAMP pathway is indeed activated (Krüger et al. 1998). However, in contrast to $\Delta u b c 1$ strains, $g p a 3_{Q 206 L}$ strains are fully pathogenic and produce tumors. Furthermore, strains expressing gpa $3_{Q 206 L}$ display a colony morphology different from wild type, termed glossy (Regenfelder et al. 1997). In this study, we show that fungal development is severely compromised in tumors induced by the combination of compatible $\mathrm{gpa}_{Q 206 L}$ strains and describe a particular $u b c l$ mutant that has a phenotype closely resembling that of gpa $_{Q 206 L}$ strains. Despite the severe reduction in fungal mass, tumors are produced. However, these tumors differ from tumors induced by wild-type strains in morphology, which points to interference in pathogen-host communication. 


\section{RESULTS}

The $\operatorname{gpa}_{Q 206 L}$ allele affects fungal development during the biotrophic phase.

Compatible gpa $3_{Q 206 L}$ strains induce tumors comparable in size to tumors induced by wild-type strains with an efficiency about twofold reduced, compared with wild type (Regenfelder et al. 1997). However, in such tumors, the characteristic darkening of the tumor due to the formation of black teliospores does not take place (Fig. 1), even when the infected plants are kept for extended periods of time (up to 6 weeks) (Fig. 1C). At this time, tumors caused by infections with wild-type strains have usually disintegrated and released the black teliospores or the host plant has died (data not shown). Another prominent feature of the tumors induced by $\mathrm{gpa}_{\text {Q206L }}$ strains is that the plant tissue stays hard and green and does not soften as in tumors caused by wildtype strains. In addition, about $20 \%$ of these tumors develop small, shootlike structures that, however, rarely extend beyond $5 \mathrm{~mm}$ (Fig. 1B and C). When tumors induced by wild-type strains are sliced, nests of dark teliospores embedded in hypertrophied plant material are visible (Fig. 1D). In tumors caused by gpa $3_{Q 206 L}$ strains, teliospores could not be detected (Fig. 1E). In mixed infection of wild-type and $g p a 3_{Q 206 L}$ strains, significantly fewer spores develop, compared with an infection with two compatible wild-type strains and these are located in the outer layers of the tumor only (Fig. 1F). These observations show that the gpa $3_{Q 206 L}$ allele causes distinct alterations of fungal development within the plant and also affects the morphology of the tumor.
The $\mathrm{gpa}_{\text {Q206L }}$ allele leads to an arrest of fungal proliferation within the tumor.

To follow stages of fungal proliferation within the infected plant tissue, we quantified fungal proliferation by preparing DNA from tumors at various times postinfection. Under the conditions employed, tumors became visible between days 8 and 10 after inoculation. The DNA was analyzed by Southern blot and was hybridized with a fungal gene, prfl. Fungal DNA could be detected in wild-type tumors from day 12 postinfection in two separate experiments (Fig. 2). During the following 9 days, the signal intensity increased, which is indicative of an increase in fungal mass (although the relative amount of fungal material within tumors harvested at a given time point may differ). In tumor tissue induced by compatible gpa $3_{Q 206 L}$ strains, a barely detectable prfl signal was seen in one tumor harvested at day 15 , while in older tumors, no signal was visible (Fig. 2). This illustrates that fungal proliferation in tumors induced by $g p a 3_{Q 206 L}$ strains is strongly reduced, compared with tumors caused by wild-type strains.

Microscopic analysis of the infection process revealed that the early steps of infection, such as appressorium formation (data not shown), growth through epidermal cells (Fig. 3A and F), and formation of branched hyphae (Fig. 3B and G), are comparable in both strains. After 7 days, both strains form branched hyphae with lobed tips (Fig. 3C and H). These stages have been described before (Banuett and Herskowitz 1996). However, the next stage, the rounding and swelling of cells observed after 9 days in infections with wild-type strains,

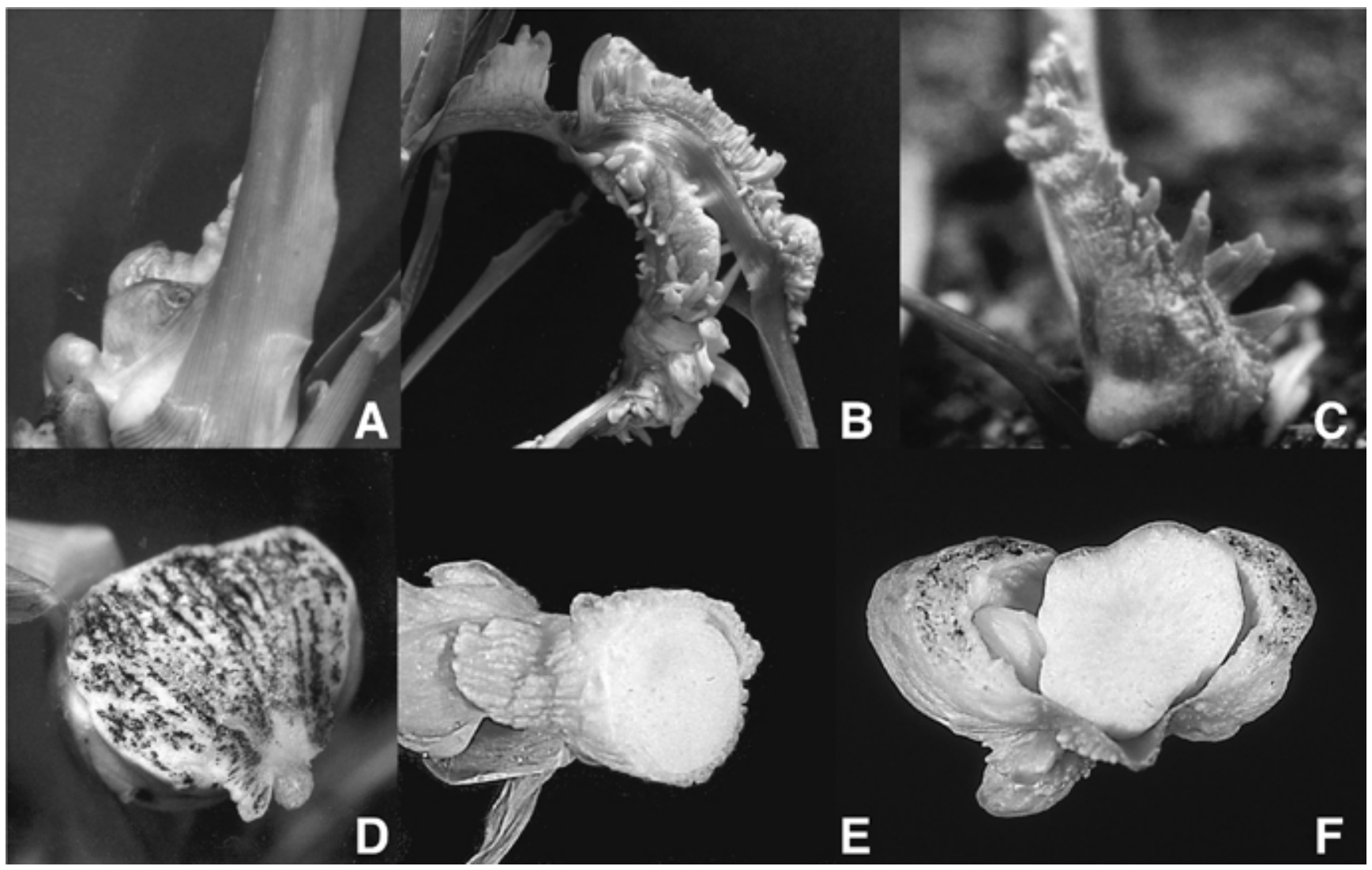

Fig. 1. Morphology of tumors caused by wild-type and compatible combinations of gpa3Q206L strains. A and D, Tumors caused by infection with FB1 and FB2 wild-type strains. B, C, and E, Tumors caused by combination of FB1 gpa3Q206L and FB2gpa3Q206L strains. F, Tumor caused by the mixture of FB1 and FB2gpa3Q206L strains. Tumors were photographed 18 days postinfection, except for $\mathbf{C}$; that photograph is 6 weeks postinfection. 
did not take place in infections by compatible gpa $3_{Q 206 L}$ strains (Fig. 3, compare D and I). By 11 days after infection, when the wild type already forms the first spores, in tumors caused by $g p a 3_{Q 206 L}$ strains hyphae remained at the stage of lobed tips (Fig. 3E and J) even after 5 weeks (data not shown). This demonstrates that the failure to detect an increase of fungal mass in tumors caused by gpa $_{\text {Q206L }}$ strains is due to a specific arrest of fungal development.

\section{gpa $_{Q 206 L}$ strains differ from wild type}

in colony morphology as well as cell surface structure.

In the following, we analyzed the morphology of mutants carrying the $\operatorname{gpa}_{Q 206 L}$ allele in more detail, compared with wild-type strains and strains carrying a deletion in $u b c l$, the gene coding for the regulatory subunit of the PKA. It has been reported earlier that colonies of $g p a 3_{Q 206 L}$ appear glossy on plates and differ from wild-type strains in this respect (Regenfelder et al. 1997). When grown on potato dextrose (PD) medium, FB1 wild-type colonies appeared dull, FB1 gpa $_{Q_{Q 206 L}}$ colonies displayed a glossy surface, and FB1 $\Delta u b c l$ showed an inter-

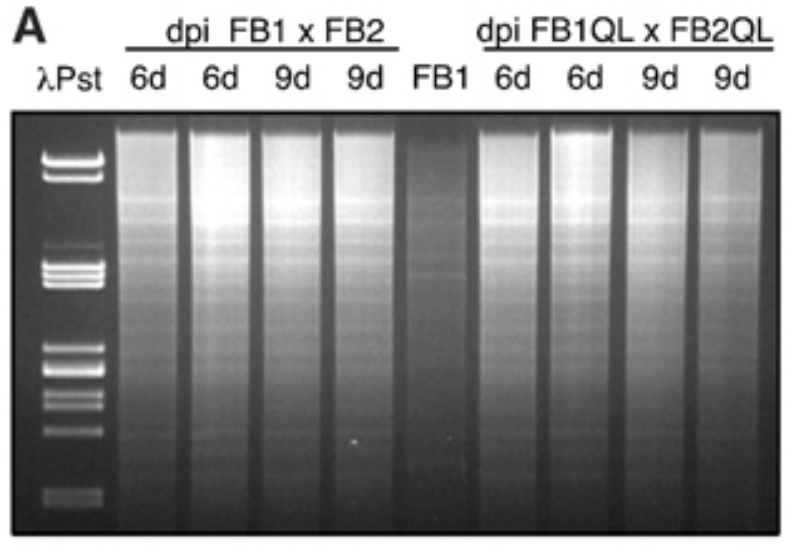

B

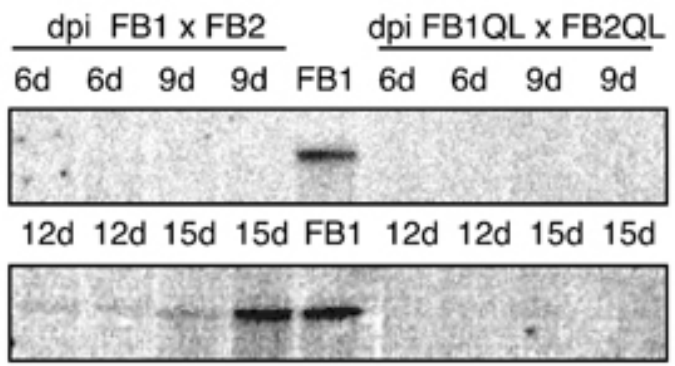

18d 18d 21d 21d FB1 18d 18d 21d 21d

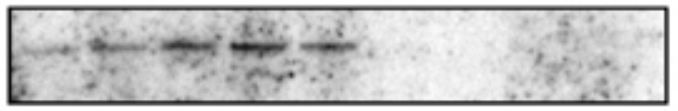

Fig. 2. Fungal proliferation in tumor tissue is strongly attenuated in tumors induced by gpa3Q206L strains. Total DNA was prepared of tumor material at indicated times after infection with a mixture of FB1 and FB2 or FB1 gpa3Q206L and FB2 gpa3Q206L strains. At each time point two different tumors were harvested and approximately $4 \mu \mathrm{g}$ of DNA was loaded side by side. As control for amount of fungal material, $2 \mu \mathrm{g}$ of DNA of strain FB1 grown in YEPS medium (10 $\mathrm{g}$ of yeast extract, $4 \mathrm{~g}$ of trypton, and $4 \mathrm{~g}$ of sucrose, per liter; modified after Tsukuda et al. 1988) was used. DNA was digested with BamHI and separated on $1 \%$ agarose gels. A, Gel representing upper blot in $\mathbf{B}$ is shown by ethidium bromide staining. B, After transfer to nitrocellulose membrane, blots were probed with a 6-kb BamHI fragment of prfl. dpi = days postinfection. mediate phenotype (Fig. 4A-C). In morphology, single cells of FB1 and FB1 1 pa $3_{Q 206 L}$ showed the same axial budding pattern (data not shown), while FB1 $\Delta u b c l$ displayed a multiple budding pattern (Gold et al. 1994). When analyzed by transmission electron microscopy, however, FB1 1 gpa $3_{Q 206 L}$ cells could be seen to be surrounded by a layer of fuzzy material that has a thickness of up to $200 \mathrm{~nm}$, while FB1 cells appear to lack this substance (Fig. 5A and B). For three-dimensional characterization of surface structure, cells were investigated by high-resolution scanning electron microscopy. While colonies of FB1 consisted of aggregates of single cells, which appear naked, in a colony of FB 1 gpa $3_{Q 206 L}$, cells are connected by a matrix like a spider web (Fig. 5C and D). Higher magnification reveals that this matrix consists of a dense network of fibrils that surround the cells, with the individual fibril being in the range of $3 \mathrm{~nm}$ in diameter (Fig. 5E and F). FB1 wildtype cells were surrounded by significantly smaller amounts of these structures (Fig. 5E), while strain FB1 $\Delta u b c 1$ also showed a high density of fibrils, comparable in amount to strain FB1 gpa $3_{Q 206 L}$ (Fig. 5G and F). The correlation between glossiness on plates and production of this matrix makes it likely that these phenotypes are linked and rely on an activated cAMP cascade.

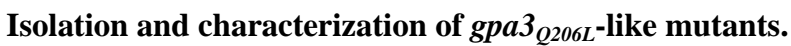

Due to the relative positions of Gpa3 and Ubc1 in the cAMP pathway, it may be expected that the deletion of $u b c l$ leads to a stronger activation of the cAMP pathway than the $g p a 3_{Q 206 L}$ mutation, and this could explain the differences observed between these mutant strains. Alternatively, Gpa3 could regulate a second signaling pathway in addition to the cAMP pathway or, in another scenario, not all of the cAMP-dependent protein kinases may be subject to regulation by Ubc1. To test these hypotheses, we screened for mutants exhibiting characteristics of gpa $_{Q 206 L}$ strains. As criteria, we used the glossy phenotype and the ability to induce tumors comparable to the tumors obtained after infection with compatible gpa $3_{Q 206 L}$ strains. About 200,000 colonies of strain FB1 were screened after UV mutagenesis (see Materials and Methods) and 36 strains with a stable glossy phenotype were obtained. The mutant strains were designated FB1gql (for glossy like gpa $3_{Q 206 L}$ ). Among these mutants, 14 strains with the strongest glossy phenotype were investigated further. A single colony of FB1 gqll is depicted in Figure $4 \mathrm{D}$. The mutations were introduced into an $a 2 b 2$ genetic background by crossing the respective FB1 $g q l$ strains with FB2. Compatible strains harboring the same $g q l$ mutation were coinjected into maize seedlings, and tumor formation and morphology were scored. Surprisingly, six of the mutants induced anthocyanin production but failed to produce tumors. This phenotype is reminiscent of $u b c l$ deletion strains (Gold et al. 1997). Five other mutants produced symptoms comparable to those of an infection with wild-type strains and three strains carrying the mutations gqll, gql4, and gql45, respectively, induced tumors comparable to infection with $\mathrm{gpa}_{\mathrm{Q} 206 \mathrm{~L}}$ strains (data not shown). The latter three mutants fulfilled the criteria set for the screen. Transformation of FB1 gqll protoplasts with a wild-type library constructed in the pCM54 selfreplicating plasmid (Bauchwitz and Hollomann 1990) allowed the isolation of a complementing gene. The phenotypes of FB1 gql4 and FB1 gql45 proved unstable upon transformation. Subcloning and sequence analysis of the complementing plas- 
mid for FB1gql1 identified the complementing gene as $u b c 1$. To verify that the mutation in FB1 gqll was indeed in the $u b c l$ gene, the $u b c l$ locus of FB1gqll and of the corresponding wildtype strain (FB1) was amplified by polymerase chain reaction (PCR) and two clones generated from independent PCRs were sequenced for each allele. A single-point mutation was identified when comparing the $u b c 1$ genes from FB1 and FB1 gql strains. It leads to an amino acid substitution from arginine (CGG) to glutamine (CAG) at position 321. We have consequently renamed this strain FB1 $u b c 1_{R 321 Q}$. As the phenotype of six mutants that caused only anthocyanin formation in infected plants is comparable to the phenotype induced by $\Delta u b c l$ strains (Gold et al. 1997), we also checked whether mutants of this class can be complemented with the $u b c l$ gene. The three mutants analyzed, FB1 gql33, FB1 gql49, and FB1 gql50, could all be complemented by $u b c l$. PCR and sequence analyses revealed that the same mutation converting the serine codon 170 (TCG) to leucine (TTG) had occurred in all three cases. This position has been described as the site where autophosphorylation of Ubc1 could occur (Gold et al. 1994). Closer examination of the phenotype induced by the $u b c 1_{R 321 Q}$ mutation revealed that the FB1 $u b c 1_{R 321 Q}$ cells display a multiple budding phenotype like cells of FB1 $\Delta u b c l$ (data not shown). Scanning electron microscopy revealed that $\mathrm{FB} 1 u b c 1_{R 321 Q}$ has a phenotype comparable to those of FB1 1 pa $3_{Q 206 L}$ and FB1 $1 u b c 1$ in terms of production of the extracellular matrix material (Fig. 5H). Plant infections demonstrated that the rate of tumor formation was attenuated in compatible combinations of $u b c 1_{R 321 Q}$ mutant strains, resulting in tumor formation in $12 \%$ of the infected plants while gpa $_{Q 206 L}$ strains form tumors in approximately $50 \%$ of the infected plants. Taken together, these data suggest that the activation of the cAMP pathway due to the $u b c 1_{R 321 Q}$ mutation may be intermediate between the levels of activation provoked by gpa $_{Q 206 L}$ and $\Delta u b c 1$ mutations and that the resulting phenotypes are direct consequences of alterations in cAMP signaling.

\section{DISCUSSION}

In this work, we present evidence that $U$. maydis mutants in which the cAMP pathway is activated secrete a capsulelike substance and have a strong defect in proliferation during the biotrophic phase. Nevertheless, such mutant strains induce plant tumors that, interestingly, differ in morphology from those induced by wild-type strains.

The cAMP pathway in $U$. maydis has been analyzed in quite some detail and it has been shown before that it plays a crucial role during pathogenic development. Of particular interest in the context of the data presented here are $u b c 1 \mathrm{mu}-$ tants, which lack the regulatory subunit of the PKA and should consequently have a fully active PKA. For such mutant strains, it has been shown that pathogenicity is severely compromised. Colonization of the plant tissue is observed and disease symptoms such as chlorosis and anthocyanin formation occur; however, tumor development is not initiated (Gold et al. 1997). With respect to these traits, gpa ${ }_{Q 206 L}$ mutants in which the cAMP pathway should also be constitutively active behave differently; most notably, they form tumors. We consider this most likely to result from different levels of activation of the PKA in the $u b c l$ and $g p a 3_{Q^{2} 26 L}$ mutant strains. First, the $g p a 3_{Q 206 L}$ mutation is supposed to reduce the GTPase activity of a $\mathrm{G} \alpha$ subunit, and in Saccharomyces cerevisiae it has been shown that the respective mutation reduces the GTPase activity more than 100-fold. However, this does not result in full activation of the $\mathrm{G} \alpha$ subunit (Masters et al. 1989). Second, the observed difference between $g p a 3_{Q 206 L}$ and $\Delta u b c 1$ strains could be due to feedback regulation of the cAMP level by active PKA, a regulatory mechanism known in $S$. cerevisiae (Nikawa et al. 1987; Ma et al. 1999; Pan and Heitman 1999). cAMP signaling in strains that express an activated $\mathrm{G} \alpha$ subunit can be down-regulated by feedback mechanisms, while strains in which the regulatory subunit of PKA is deleted would escape such control. This hypothesis is supported by our finding that a $u b c 1$ mutant strain $\left(\mathrm{FB} 1 u b c 1_{R 321 Q}\right)$ could be identified that displayed phenotypes similar to those of the $g p a 3_{Q 206 L}$ strain. The $u b c l$ mutant allele of this strain carries a single amino acid substitution from arginine to glutamine. It does not involve a change in any characterized motif and we suspect that it affects protein conformation in such a way as to lead to a partially functional protein. Thus, in contrast to deletion mutants, a $u b c 1_{R 321 Q}$ strain expresses a regulatory subunit that

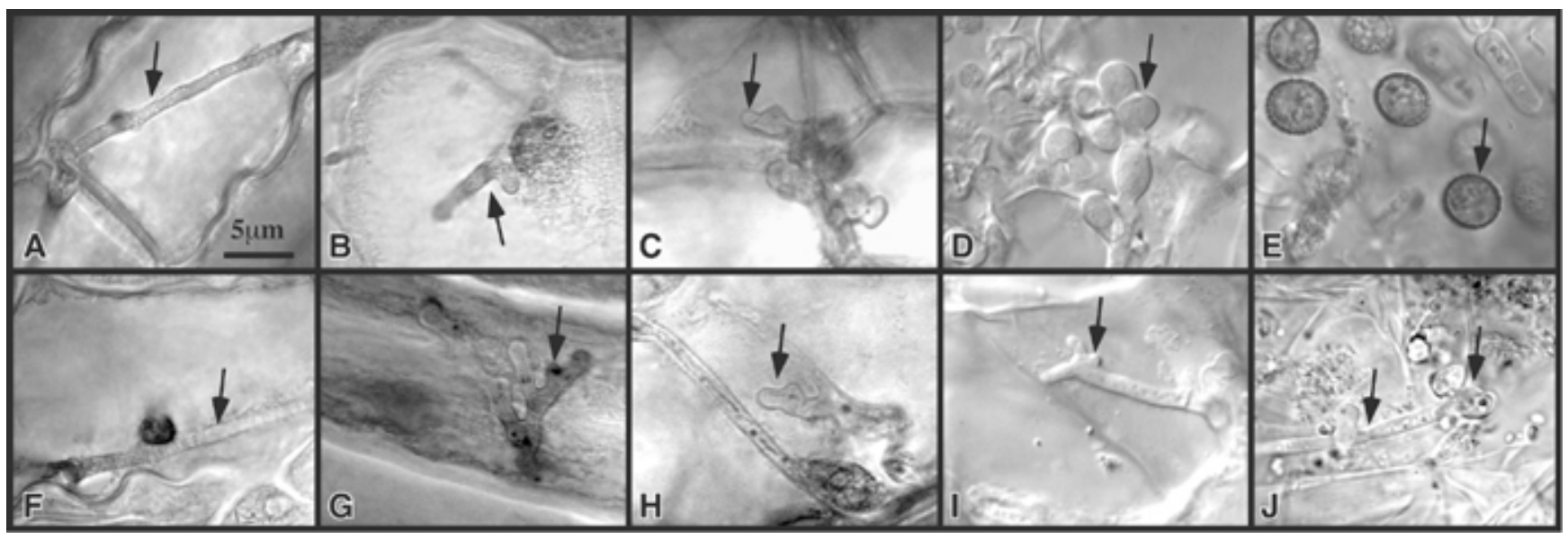

Fig. 3. Development of gpa3Q206L strains is arrested at stage of lobed hyphae. Plants were infected with A-E, a mixture of FB1 and FB2; or F-J, FB1 gpa 3 Q206L and FB2gpa3Q206L strains. Leaves were harvested $\mathbf{A}$ and F, 3; $\mathbf{B}$ and $\mathbf{G}, 5$; or $\mathbf{C}$ and $\mathbf{H}, 7$ days after infection and stained with aniline blue according to Gold et al. (1997). For samples at $\mathbf{D}$ and I, 9 days after infection; and $\mathbf{E}$ and $\mathbf{J}, 11$ days after infection, tumors on leaves were cut with a razor blade and sections were examined without further staining. 
could still function in feedback regulation. Unfortunately, attempts to determine free PKA activities in whole cell extracts of the various strains failed because the amounts were too low to be detected by the PepTag assay (Promega, Madison, WI, U.S.A.) (data not shown).

In the screen for $\mathrm{gpa}_{\mathrm{Q}^{206 L}}$-like mutants, three independent mutants were identified that carried the same mutation in $u b c l$, $u b c 1_{S 170 L}$. This mutation is at the presumed autophosphorylation site, which was used to classify $u b c l$ as a type II regulatory subunit of PKA (Gold et al. 1994; Taylor et al. 1990). Autophosphorylation is supposed to facilitate dissociation from the catalytic subunit (Taylor et al. 1990). In the yeast homologue $B C Y 1$, the effects of different amino acid substitutions at this residue are described. When the serine is exchanged by a smaller amino acid (Ala, Gly) the resulting protein is a better inhibitor of the catalytic subunit. However, if other amino acids are introduced at this position (Glu, Asp, Lys, Phe), the capacity of the protein to bind the catalytic subunit is weakened. The authors conclude that it is probably the size of the amino acid at the autophosphorylation site that leads to gain or loss of function (Kuret et al. 1988). Based on these data, we would predict that the $u b c 1_{S 170 L}$ allele encodes a protein that shows only weak binding to Adr1. In summary, we identified a series of $u b c l$ alleles that differ in their regulatory activities, as reflected by their pathogenic potential. Strains carrying the $u b c 1_{R 321 Q}$ allele are able to form tumors (but less so than $\left.g p a 3_{Q 206 L}\right)$ while strains carrying $u b c 1_{S 170 L}$ do not and thus behave like null mutants.

Electron microscopy has revealed that the activation of the cAMP pathway by various mutations leads to coating of the cells with an unidentified fibrillous material, which is present in the wild type also, but at much lower abundance. This correlates with a glossy colony surface of the mutants activated for the cAMP cascade, which can also be induced by addition

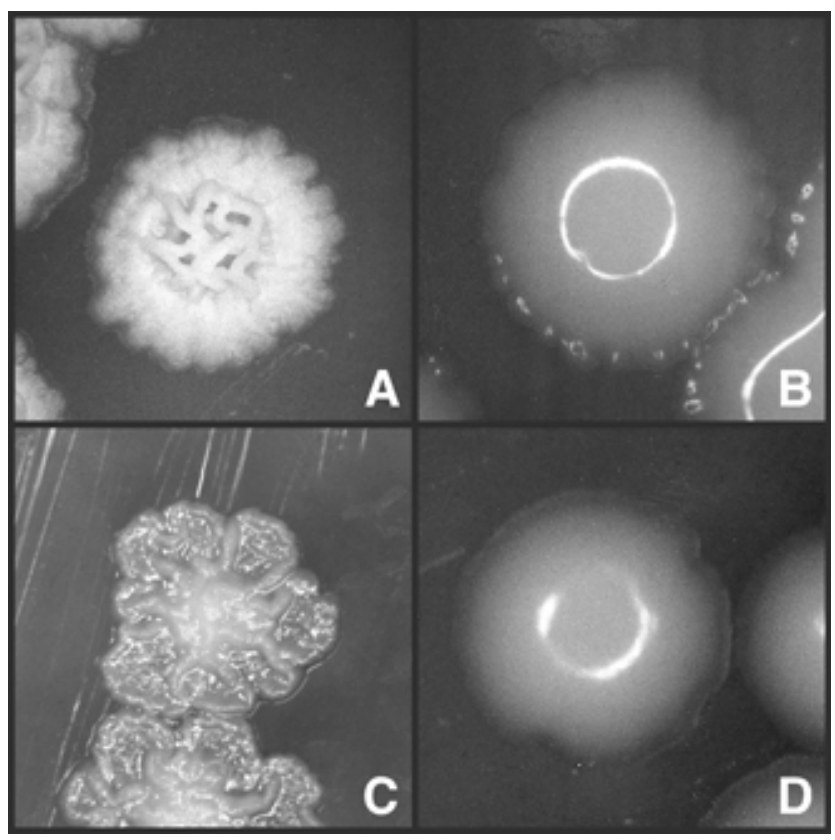

Fig. 4. Colony morphology of strains with an activated cyclic AMP (cAMP) cascade. Single colonies of the indicated strains were grown on potato dextrose plates and photographed after 6 days of incubation at $28^{\circ} \mathrm{C}$. A, FB1; B, FB1 gpa $3 Q 206 L$; C, FB1 $\Delta u b c 1$; and D, FB1 $u b c 1 R 321 Q$. of exogenous cAMP to wild-type strains (J. Krüger, unpublished data). A connection between capsule formation and pathogenic development has been demonstrated in another basidiomycete, Cryptococcus neoformans. Mutants in the $C$. neoformans $\mathrm{G} \alpha$ subunit Gpa1, a likely homolog of $U$. maydis Gpa3, fail to form a capsule, and this can be rescued by addition of exogenous cAMP (Alspaugh et al. 1998). Although formation of the capsule in U. maydis and C. neoformans appears to be under the same type of control, it remains to be shown whether capsule formation is linked to the aberrant tumor phenotype observed after infection with gpa $_{Q_{Q 206 L}}$ strains.

Our results reiterate that a regulated cAMP cascade is crucial for the development of $U$. maydis during the biotrophic phase, which had been demonstrated previously by the analyses of uacl, ubcl, adrl, and gpa3 knockout strains (Gold et al. 1994, 1997; Dürrenberger et al. 1998; Regenfelder et al. 1997). However, the analysis of mutants that lead to more subtle changes in the activity of the cAMP pathway has provided unexpected insights into the process of tumor development. Tight regulation of the level of cAMP signaling in a spatial and temporal manner appears to be crucial for pathogenicity. This is supported by the observation that strains in which cAMP signaling is frozen at different levels are disturbed at distinct stages of development within the plant.

Another intriguing aspect is the communication of the pathogen with the plant. The finding that tumor induction after infection with $g p a 3_{Q 206 L}$ strains is seen despite the low abundance of fungal cells in these tissues shows that efficient signaling of only a few cells is sufficient for tumor formation. In addition, a strain expressing a constitutively active $\mathrm{G} \alpha$ subunit $\mathrm{Gpa} 3_{\mathrm{Q} 206 \mathrm{~L}}$ elicits tumors with shootlike structures. This suggests that fungal cAMP signaling affects the production of molecules that set off phytohormone balance of the adjacent plant cells implicated in tumor formation. It will be an exciting challenge to analyze with molecular and biochemical techniques the tumor material, which hardly contains fungal mass. This might provide novel insights into how the fungus communicates with the plant to elicit tumor development.

\section{MATERIALS AND METHODS}

\section{Strains, plasmids, and plasmid constructions.}

The Escherichia coli $\mathrm{K} 12$ strain DH5 $\alpha$ mcr was used as a host for plasmid amplification. The U. maydis strains FB1 (al b1) and FB2 (a2 b2) (Banuett and Herskowitz 1989), FB1 1 gpa $3_{Q 206 L}$, FB2 $g p a 3_{Q 206 L}$ (Regenfelder et al. 1997), and FB1 $\Delta u b c 1$ (Hartmann et al. 1999) have been described before. For sequencing purposes restriction fragments were cloned in pBluescript SKII+ (Stratagene, La Jolla, CA, U.S.A.).

\section{Growth conditions and media.}

Strains were grown at $28^{\circ} \mathrm{C}$ in YEPS medium ( $10 \mathrm{~g}$ of yeast extract per liter, $4 \mathrm{~g}$ of trypton per liter, and $4 \mathrm{~g}$ of sucrose per liter; modified after Tsukuda et al. 1988), in CM (Holliday 1974), or PD medium (Difco, Detroit, MI, U.S.A.). The colony morphology was observed on PD plates. Nourseothricin (nat) was purchased from the Hans-Knöll Institute (Jena, Germany). All chemicals used were of analytical grade and were obtained from Sigma (Deisenhofen, Germany) or Merck (Darmstadt, Germany). Plant infections were performed as described previously (Gillissen et al. 1992) with the maize culti- 
vars Early Golden Bantam (Olds Seeds, Madison, WI, U.S.A.) and Gaspar Flint (originally obtained from B. Burr, Brookhaven National Laboratory).

UV mutagenesis of $\boldsymbol{U}$. maydis.

FB1 was grown in YEPS liquid culture to an optical density of 1.0. Cells were plated on PD plates and UV-irradiated $(254 \mathrm{~nm})$ to a survival rate between 0.1 and $1 \%$. Surviving cells were grown for 3 days in the dark and then scored for a glossy colony morphology. Mutants were crossed with FB2 and haploid progeny strains were identified that carried the mutation conferring glossiness in the $a 2 b 2$ background by performing appropriate crosses with tester strains.

The pCM54 library (Bauchwitz and Holloman 1990) was used for complementation of strain FB1gqll. The complementing plasmid obtained was subcloned in the self replicating plasmid pKurt (F. Schauwecker and R. Kahmann, unpublished data) and a 3.8-kb XbaI fragment was shown to retain complementing activity.

\section{DNA and RNA procedures.}

Standard molecular techniques followed Sambrook et al. (1989). Transformation of U. maydis followed published procedures (Schulz et al. 1990). U. maydis DNA was prepared according to Hoffman and Winston (1987). Tumor DNA was prepared from $2 \mathrm{~g}$ of plant material, which was ground in liquid nitrogen and subsequently extracted with $5 \mathrm{ml}$ of cetyltriethylammonium bromide (CTAB) buffer (2\% CTAB in 0.1 $\mathrm{M}$ Tris-HCL, $\mathrm{pH} 7.5$, and $0.7 \mathrm{M} \mathrm{NaCl}$ ) at $65^{\circ} \mathrm{C}$ for $20 \mathrm{~min}$. After chloroform extraction, the supernatant was precipitated with isopropanol, washed with $70 \%$ ethanol, and resuspended in $100 \mu \mathrm{l}$ of Tris-EDTA (TE) buffer. Southern filters were probed with a 6-kb Bam HI fragment of prfl isolated from plasmid pRF6.0B (Hartmann et al. 1999). A STORM Phosphor Imager (Molecular Dynamics, Sunnyvale, CA, U.S.A.) was used for signal detection.

PCR amplification and analysis of the $u b c 1$ mutant alleles.

Standard buffer conditions for Taq DNA polymerase were used for amplification in a final volume of $50 \mu \mathrm{l}$. About $40 \mathrm{ng}$ of genomic DNA and 25 pmol of the primers GQ11-1 (5'GCGACCCTTTTTTCGGCTTGGCG-3') and GQL1-2 (5'ACCATCCAAAGCAGGCGCACT-GG-3') were added to the reaction. Following an initial denaturation step of $94^{\circ} \mathrm{C}$ for 4 min, 30 cycles of $94^{\circ} \mathrm{C}$ for $1 \mathrm{~min}, 61^{\circ} \mathrm{C}$ for $1 \mathrm{~min}$, and $72^{\circ} \mathrm{C}$ for $2 \mathrm{~min}$ were performed, followed by a final 4-min incubation at $72^{\circ} \mathrm{C}$. PCR products were cloned with the TOPO TA cloning kit (Invitrogen; Groningen, the Netherlands) according to instructions by the manufacturer. Universal, reverse, and internal primers were used to generate double-stranded sequences of the $u b c l$ mutant alleles. Sequences were determined with an ABI 373 automated sequencer.

The wild-type sequence of $u b c l$ determined from strain FB1 differs from the sequence previously published (accession no. L33917). At codon 44 the triplet GCG is changed to GAG leading to a substitution of A by $\mathrm{E}$, two deletions of three bases each affect codon position 78-80 (CCC TCA GCG to CCT CAG) and codon position 424-426 (GCG CCT CGC to CGC TGC), respectively. In both ,cases this leads to a deletion and a concomitant change in amino acids (PSA to PQ and APR to RC, respectively). In addition, we detect the dele- tion of three bases between codons 461 and codon 493 leading to alterations in amino acid sequence over a region of 33 amino acids.

\section{Light microscopy.}

$U$. maydis and maize tumors containing different developmental stages of $U$. maydis were microscopically analyzed with a Zeiss Axiophot with differential interference contrast (DIC) optics. The aniline blue staining of the fungal hyphae in planta was as described by Gold et al. (1997). The pictures were taken in HiPic with a Hamamatsu CCD camera and processed by Image Pro. Colonies were magnified with an Olympus Binocular for documentation.

\section{Electron microscopy.}

Strains were grown on PD plates for 3 days at $28^{\circ} \mathrm{C}$. Cells were fixed with $2.5 \%$ (vol/vol) glutaraldehyde in $75 \mathrm{mM}$ ca-

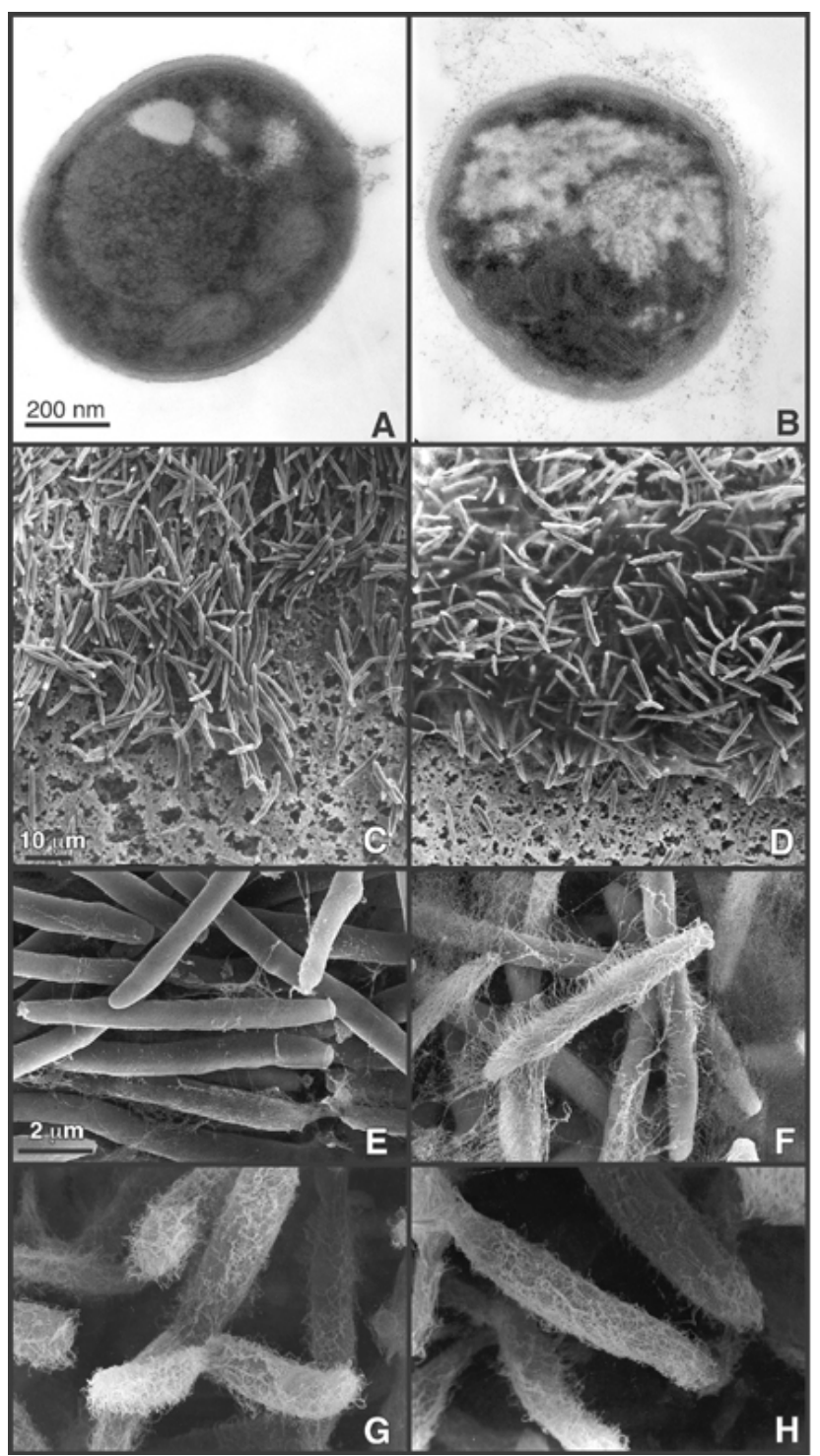

Fig. 5. Strains with activated cyclic AMP (cAMP) cascade surrounded by a capsulelike material. $\mathbf{A}$ and $\mathbf{B}$, Transmission electron micrographs. C-H, Scanning electron micrographs. A, C, and E, Strain FB1. B, D, and F, Strain FB1 $g p a 3 Q 206 L$. G, FB1 $\Delta u b c 1$. H, FB1 $u b c 1 R 321 Q$. 
codylate buffer ( $\mathrm{pH} 7.0)$, postfixed with osmium tetroxide, dehydrated in a graded series of acetone solutions, infiltrated and embedded in Spurr's lox viscosity resin. Ultrathin sections were examined in a Zeiss EM 912 transmission electron microscope equipped with an integrated energy filter, operated at $80 \mathrm{kV}$ in the zero loss mode.

For scanning electron microscopy, dehydrated cells were critical point-dried from liquid $\mathrm{CO}_{2}$, mounted on stubs, and coated with 3 to $5 \mathrm{~nm}$ of platinum with a magnetron sputter coater. The specimens were examined with a Hitachi S-4100 field emission scanning electron microscope operated at $8 \mathrm{kV}$.

\section{ACKNOWLEDGMENTS}

We thank Karl Heinz Braun for expert technical help. This work was supported by the Sonderforschungsbereich 369 of the Deutsche Forschungsgemeinschaft. G. L. was supported by a Marie Curie postdoctoral training grant from the European Commission.

\section{LITERATURE CITED}

Alspaugh, J. A., Perfect, J. R., and Heitman, J. 1998. Signal transduction pathways regulating differentiation and pathogenicity of Cryptococcus neoformans. Fungal Gen. Biol. 25:1-14.

Banuett, F. 1995. Genetics of Ustilago maydis, a fungal pathogen that induces tumors in maize. Annu. Rev. Genet. 29:179-208.

Banuett, F., and Herskowitz, I. 1989. Different $a$ alleles of Ustilago maydis are necessary for maintenance of filamentous growth but not for meiosis. Proc. Natl. Acad. Sci. U.S.A. 86:5878-5882.

Banuett, F., and Herskowitz, I. 1996. Discrete developmental stages during teliospore formation in the corn smut fungus, Ustilago maydis. Genes Dev. 122:2965-2976.

Bauchwitz, R., and Holloman, W. K. 1990. Isolation of the rec2 gene controlling recombination in Ustilago maydis. Gene 96:285-288.

Bölker, M., Urban, M., and Kahmann, R. 1992. The $a$ mating type locus of $U$. maydis specifies cell signalling components. Cell 68:441-450.

Dürrenberger, F., Wong, K., and Kronstad, J. W. 1998. Identification of a cAMP-dependent protein kinase catalytic subunit required for virulence and morphogenesis in Ustilago maydis. Proc. Natl. Acad. Sci. U.S.A. 95:5684-5689.

Gillissen, B., Bergemann, J., Sandmann, C., Schroeer, B., Bölker, M., and Kahmann, R. 1992. A two-component regulatory system for self/ non-self recognition in Ustilago maydis. Cell 68:1-20.

Gold, S., Duncan, G., Barrett, K., and Kronstad, J. 1994. cAMP regulates morphogenesis in the fungal pathogen Ustilago maydis. Genes Dev. 8:2805-2816.

Gold, S. E., Brogdon, S. M., Mayorga, M. E., and Kronstad, J. W. 1997. The Ustilago maydis regulatory subunit of a cAMP-dependent protein kinase is required for gall formation in maize. Plant Cell 9:1585-1594.

Hartmann, H. A., Kahmann, R., and Bölker, M. 1996. The pheromone response factor coordinates filamentous growth and pathogenic development in Ustilago maydis. EMBO J. 15:1632-1641.
Hartmann, H. A., Krüger, J., Lottspeich, F., and Kahmann, R. 1999. Environmental signals controlling sexual development of the corn smut fungus Ustilago maydis through the transcriptional regulator Prf1. Plant Cell 11:1293-1306.

Hoffman, C. S., and Winston, F. 1987. A ten-minute DNA preparation from yeast efficiently releases autonomous plasmids for transformation in E. coli. Gene 57:267-272.

Holliday, R. 1974. Ustilago maydis. Pages 575-595 in: Handbook of Genetics. Vol. 1. R. C. King, ed. Plenum, New York, U.S.A.

Kämper, J., Reichmann, M., Romeis, T., Bölker, M., and Kahmann, R. 1995. Multiallelic recognition: Nonself-dependent dimerization of the $\mathrm{bE}$ and $\mathrm{bW}$ homeodomain proteins in Ustilago maydis. Cell 81:73-83.

Krüger, J., Loubradou, G., Regenfelder, E., Hartmann, H. A., and Kahmann, R. 1998. Crosstalk between cAMP and pheromone signaling pathways in Ustilago maydis. Mol. Gen. Genet. 260:193-198.

Kuret, J., Johnson, K. E., Nicolette, C., and Zoller, M. J. 1988. Mutagenesis of the regulatory subunit of yeast cAMP-dependent protein kinase. J. Biol. Chem. 263:9149-9154.

Ma, P., Wera, S., Van Dijck, P., and Thevelein, J. M. 1999. The PDE1 encoded low-affinity phosphodiesterase in the yeast Saccharomyces cerevisiae has a specific function in controlling agonist-induced cAMP signalling. Mol. Biol. Cell 10:91-104.

Masters, S. B., Miller, R. T., Chi, M.-H., Chang, F.-H., Biedermann, B., Lopez, N. G., and Bourne, H. R. 1989. Mutations in the GTP-binding site of $\mathrm{G}_{\mathrm{s} \alpha}$ alter stimulation of adenylyl cyclase. J. Biol. Chem. 264: 15467-15474.

Müller, P., Aichinger, C., Feldbrügge, M., and Kahmann, R. 1999. The MAP kinase Kpp2 regulates mating and pathogenic development in Ustilago maydis. Mol. Microbiol. 34:1007-1017.

Nikawa, J., Cameron, S., Toda, T., Ferguson, K. W., and Wigler, M. 1987. Rigorous feedback control of cAMP levels in Saccharomyces cerevisiae. Genes Dev. 1:931-937.

Pan, X., and Heitman, J. 1999. Cyclic AMP-dependent protein kinase regulates pseudohyphal differentiation in Saccharomyces cerevisiae. Mol. Cell. Biol. 19:4874-4887.

Regenfelder, E., Spellig, T., Hartmann, A., Lauenstein, S., Bölker, M., and Kahmann, R. 1997. G proteins in Ustilago maydis: Transmission of multiple signals? EMBO J. 16:1934-1942.

Sambrook, J., Fritsch, E. F., and Maniatis, T. A. 1989. Molecular Cloning: A Laboratory Manual. 2nd ed. Cold Spring Harbor Laboratory, Cold Spring Harbor, NY, U.S.A.

Schulz, B., Banuett, F., Dahl, M., Schlesinger, R., Schäfer, W., Martin, T., Herskowitz, I., and Kahmann, R. 1990. The $b$ alleles of Ustilago maydis, whose combinations program pathogenic development, code for polypeptides containing a homeodomain-related motif. Cell 60: 295-306.

Spellig, T., Bölker, M., Lottspeich, F., Frank, R. W., and Kahmann, R. 1994. Pheromones trigger filamentous growth in Ustilago maydis. EMBO J. 13:1620-1627.

Taylor, S. S., Buechler, J. A., and Yonemoto, W. 1990. cAMP-dependent protein kinase: Framework for a diverse family of regulatory enzymes. Annu. Rev. Biochem. 59:971-1005.

Tsukuda, T., Carleton, S., Fotheringham, S., and Holloman, W. K. 1988. Isolation and characterisation of an autonomously replicating sequence from Ustilago maydis. Mol. Cell. Biol. 8:3703-3709. 